\title{
A New Direct Method for Solving Nonlinear Volterra-Fredholm-Hammerstein Integral Equations via Optimal Control Problem
}

\author{
M. A. El-Ameen ${ }^{1}$ and M. El-Kady ${ }^{2}$ \\ ${ }^{1}$ Faculty of Engineering, Umm Al-Qura University, P.O. Box 5555, Mecca, Saudi Arabia \\ ${ }^{2}$ Faculty of Science, Helwan University, Helwan, Egypt
}

Correspondence should be addressed to M. A. El-Ameen, kearmossa@yahoo.com

Received 28 December 2011; Accepted 28 January 2012

Academic Editor: Shuyu Sun

Copyright (c) 2012 M. A. El-Ameen and M. El-Kady. This is an open access article distributed under the Creative Commons Attribution License, which permits unrestricted use, distribution, and reproduction in any medium, provided the original work is properly cited.

A new method for solving nonlinear Volterra-Fredholm-Hammerstein (VFH) integral equations is presented. This method is based on reformulation of VFH to the simple form of Fredholm integral equations and hence converts it to optimal control problem. The existence and uniqueness of proposed method are achieved. Numerical results are given at the end of this paper.

\section{Introduction}

The nonlinear integral equations arise in the theory of parabolic boundary value problems, engineering, various mathematical physics, and theory of elasticity [1-3]. In recent years, several analytical and numerical methods of this kind of problems have been presented $[4,5]$. Analytically, the decomposition methods are used in $[6,7]$. The classical method of successive approximations was introduced in [8], while some kind of appropriate projection such as Galerkin and collocation methods have been applied in [9-13]. These methods often transform integral or integrodifferential equations into a system of linear algebraic equations which can be solved by direct or iterative methods. In [14], the authors used Taylor series to solve the following nonlinear Volterra-Fredholm integral equation:

$$
y(x)=f(x)+\lambda_{1} \int_{0}^{x} k_{1}(x, t)[y(t)]^{p} d t+\lambda_{2} \int_{0}^{1} k_{2}(x, t)[y(t)]^{q} d t, \quad p, q \in R
$$


whereas the Legendre wavelets method for a special type was applied in [15] for solving the nonlinear Volterra-Fredholm integral equation of the form

$$
y(x)=f(x)+\lambda_{1} \int_{0}^{x} k_{1}(x, t)[F(y(t))] d t+\lambda_{2} \int_{0}^{1} k_{2}(x, t) G(y(t)) d t
$$

where $f(x)$ and the kernels $k_{1}(x, t)$ and $k_{2}(x, t)$ are assumed to be in $L^{2}(R)$ on the interval $0 \leq x, t \leq 1$. The nonlinear Volterra-Fredholm-Hammerstein integral equation is given in [16] as follows:

$$
y(t)=f(t)+\lambda_{1} \int_{0}^{t} k_{1}(t, s)\left[g_{1}(s, y(s))\right] d s+\lambda_{2} \int_{0}^{1} k_{2}(t, s) g_{2}(s, y(s)) d s, \quad 0 \leq t, s \leq 1 .
$$

In this paper, we introduce a method to find the numerical solution of a nonlinear VolterraFredholm-Hammerstein integral equation of the form:

$$
\phi(t)=f(t)+\lambda_{1} \int_{0}^{t} V(t, s, \phi(s)) d s+\lambda_{2} \int_{a}^{b} F(t, s, \phi(s)) d s, \quad 0 \leq t, s \leq 1,
$$

where $f(t), V(t, s, \phi(s))$, and $F(t, s, \phi(s))$ are assumed to be in $L^{2}(R)$ and satisfy the Lipschitz condition

$$
\left|K\left(t, s, \phi_{1}(s)\right)-K\left(t, s, \phi_{2}(s)\right)\right| \leq N(t, s)\left|\phi_{1}(s)-\phi_{2}(s)\right|
$$

This paper is organized as follows. In Section 2, we present a form of (1.4) by Fredholm type integral equation, which can convert it into optimal control problem (OC). In Section 3, the existence and uniqueness are presented. The computational results are shown in Section 4.

\section{Problem Reformulation}

Let the VFH given in (1.4) be written in the form

$$
\phi(t)=f(t)+\lambda \int_{a}^{b} k(t, s, \phi(s)) d s,
$$

such that

$$
\begin{gathered}
k(t, s, \phi(s))=G(t, s, \phi(s))+F(t, s, \phi(s)), \\
G(t, s, \phi(s))=e(t, s, \phi(s)) V(t, s, \phi(s)), \\
e(t, s, \phi(s))= \begin{cases}1 & a<s<t<b, \\
0 & s>t,\end{cases}
\end{gathered}
$$


Journal of Applied Mathematics

and the kernel $k(t, s, \phi(s)) \in C[a, b] \times[a, b]$ satisfyies

$$
|k(t, s, \phi(s))| \leq M, \quad|f(t)| \leq K,
$$

where $M, K$ are arbitrary constants.

It is easy to see that (2.1) can be written as follows:

$$
\begin{aligned}
\phi(t)-f(t) & =\int_{a}^{t}[\dot{\phi}(s)-\dot{f}(s)] d s+[\phi(a)-f(a)] \\
& =\int_{a}^{b} \delta[\dot{\phi}(s)-\dot{f}(s)] d s+[\phi(a)-f(a)], \quad \text { where } \delta= \begin{cases}1 & a<s<t<b, \\
0 & s>t,\end{cases}
\end{aligned}
$$

then

$$
\int_{a}^{b} \delta[\dot{\phi}(s)-\dot{f}(s)] d s+[\phi(a)-f(a)]=\lambda \int_{a}^{b} k(t, s, \phi(s)) d s .
$$

Since

$$
\phi(a)-f(a)=\lambda \int_{a}^{b} k(a, s, \phi(s)) d t
$$

therefore,

$$
\int_{a}^{b} \delta[\dot{\phi}(s)-\dot{f}(s)] d s+\lambda \int_{a}^{b}[k(a, s, \phi(s))-k(t, s, \phi(s))] d s=0 .
$$

Let

$$
G(t)=\int_{a}^{b}\{\delta[\dot{\phi}(s)-\dot{f}(s)]+\lambda[k(a, s, \phi(s))-k(t, s, \phi(s))]\} d s=0,
$$

that is, if

$$
|G(t)|=0 .
$$

By integrating (2.9), we have

$$
\int_{a}^{b}|G(t)| d t=0
$$


On the other hand, one can define the following equality:

$$
F(t, s, \phi(t), u(t))=\delta[\dot{\phi}(s)-\dot{f}(s)]+\lambda[k(a, s, \phi(s))-k(t, s, \phi(s))] .
$$

This will lead us to the following inequality:

$$
\int_{a}^{b}|G(t)| d t \leq \iint_{a}^{b}|F(t, s, \phi(t), u(t))| d s d t
$$

where

$$
\dot{\phi}(s)=u(s), \quad s \in[a, b] .
$$

With the boundary conditions

$$
\phi(a)=f(a)+\int_{a}^{b} k(a, s, \phi(s)) d s, \quad \phi(b)=f(b)+\int_{a}^{b} k(b, s, \phi(s)) d s .
$$

At the end, we have the following OC problem:

minimize

$$
I=\int_{\Omega}|F(t, s, \phi(t), u(t))| d s d t
$$

subject to

$$
\dot{\phi}(s)=u(s), \quad s \in[a, b],
$$

$\phi(a)$ and $\phi(b)$ are defined in (2.14) where $\Omega=[a, b] \times[a, b]$.

The existence and uniqueness of (2.1) will be considered in the next section by using the successive approximation method.

\section{Existence and Uniqueness}

The solution $\phi(t)$ of (2.1) can be approximated successively as follows:

$$
\phi_{1}(t)-\lambda \int_{a}^{b} k\left(t, s, \phi_{0}(s)\right) d s=f(t)
$$

Thus, we obtain sequence of functions $\phi_{0}(t), \phi_{1}(t), \ldots, \phi_{n}(t)$, such that

$$
\phi_{n}(t)-\lambda \int_{a}^{b} k\left(t, s, \phi_{n-1}(s)\right) d s=f(t), \quad n \geq 1,
$$

with $\phi_{0}(t)=f(t)$. 
It is convenient to introduce

$$
\psi_{n}(t)=\phi_{n}(t)-\phi_{n-1}(t), \quad n \geq 1,
$$

with $\psi_{0}(t)=f(t)$.

Subtracting from (3.2), the same equation with replacing $n$ by $n-1$, we get

$$
\phi_{n}(t)-\phi_{n-1}(t)=\lambda \int_{a}^{b} k\left(t, s, \phi_{n-1}(s)\right) d s-\lambda \int_{a}^{b} k\left(t, s, \phi_{n-2}(s)\right) d s .
$$

Using (3.3), we have

$$
\psi_{n}(t)=\lambda \int_{a}^{b} k\left(t, s, \psi_{n-1}(s)\right) d s \quad n \geq 1
$$

Also, from (3.3), we deduce that

$$
\phi_{n}(t)=\sum_{i=0}^{n} \psi_{i}(t)
$$

The existence and uniqueness of the solution can be followed.

Theorem 3.1. If the kernel $k(t, s, \phi(s))$ and the function $f(t)$ are continuous and satisfy condition (2.3) in $a<s<t<b$, then the integral equation (2.1) possesses a unique continuous solution.

Proof. From (3.5), we get

$$
\begin{aligned}
\left|\psi_{n}(t)\right| & =\left|\lambda \int_{a}^{b} k\left(t, s, \psi_{n-1}(s)\right) d s\right| \\
& \leq \lambda\left|k\left(t, s, \psi_{n-1}(s)\right)\right| \int_{a}^{b} d s \\
& \leq \lambda(b-a) M .
\end{aligned}
$$

We now show that this $\phi(t)$ satisfies (2.1).

The series (3.6) is uniformly convergent since the term $\psi_{i}(t)$ is dominated by $\lambda(b-a) M$. Then,

$$
\begin{aligned}
\lambda \int_{a}^{b} k\left(t, s, \sum_{i=0}^{\infty} \psi_{i}(s)\right) d s & =\sum_{i=0}^{\infty} \lambda \int_{a}^{b} k\left(t, s, \psi_{i}(s)\right) d s \\
& =\sum_{i=0}^{\infty} \psi_{i+1}(t)=\sum_{i=0}^{\infty} \psi_{i+1}(t)+\psi_{0}(t)-\psi_{0}(t) .
\end{aligned}
$$


Hence, we have

$$
\lambda \int_{a}^{b} k\left(t, s, \sum_{i=0}^{\infty} \psi_{i}(s)\right) d s=\sum_{i=0}^{\infty} \psi_{i}(t)-f(t) .
$$

This proves that $\phi(t)$, defined in (3.6), satisfies (2.1). Since each of the $\psi_{i}(t)$ is clearly continuous, therefore $\phi(t)$ is continuous, where it is the limit of a uniformly convergent sequence of continuous functions.

To show that $\underset{\sim}{\phi}(t)$ is a unique continuous solution, suppose that there exists another continuous solution $\widetilde{\phi}(t)$ of (2.1), Then,

$$
\tilde{\phi}(t)-\lambda \int_{a}^{b} k(t, s, \tilde{\phi}(s)) d s=f(t) .
$$

Subtracting (3.10) from (2.1), we get

$$
\phi(t)-\tilde{\phi}(t)=\lambda \int_{a}^{b} k(t, s,\{\phi(s)-\tilde{\phi}(s)\}) d s .
$$

Since $\phi(t)$ and $\tilde{\phi}(t)$ are both continuous, there exists a constant $B$ such that

$$
|\phi(t)-\tilde{\phi}(t)| \leq B
$$

By using the condition of (2.3), the inequality (3.12) becomes

$$
|\phi(t)-\tilde{\phi}(t)| \leq \lambda(b-a) M B
$$

For the large enough $n$, the right-hand side is arbitrary small, then

$$
\phi(t)=\tilde{\phi}(t)
$$

This completes the proof.

\section{Computational Results}

In this section, some numerical experiments will be carried out in order to compare the performances of the new method with respect to the classical collocation methods. The method has been applied to the following three test problems $[16,17]$.

Example 4.1. Consider the Volterra-Fredholm-Hammerstein integral equation

$$
x(s)=2 \cos (s)-2+3 \int_{0}^{s} \sin (s-t) x^{2}(t) d t+\frac{6}{7-6 \cos (1)} \int_{0}^{1}(1-t) \cos ^{2}(s)(t+x(t)) d t .
$$

The exact solution is given by $x(s)=\cos (s)$. 
Table 1: Maximum errors for collocation and OC methods.

\begin{tabular}{lcc}
\hline$N$ & Errors in collocation method & Errors in optimal control method \\
\hline 8 & $3.17 \mathrm{E}-11$ & $2.22 \mathrm{E}-13$ \\
12 & $1.11 \mathrm{E}-12$ & $1.11 \mathrm{E}-15$ \\
16 & $4.03 \mathrm{E}-13$ & $3.33 \mathrm{E}-16$ \\
20 & $7.93 \mathrm{E}-15$ & $2.22 \mathrm{E}-16$ \\
24 & $2.22 \mathrm{E}-16$ & $2.22 \mathrm{E}-16$ \\
\hline
\end{tabular}

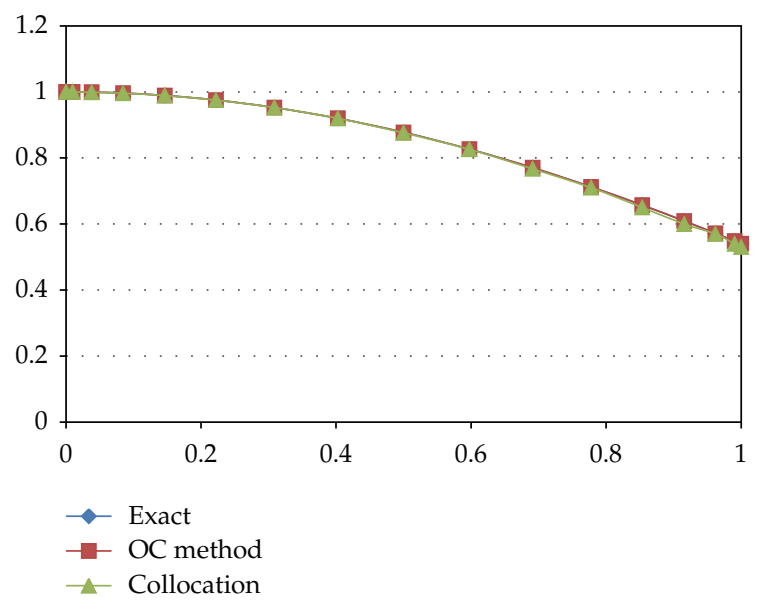

Figure 1: Observed results for Example $4.1(N=16)$.

The computational maximum absolute errors for different values of $N$ are shown in Table 1. It is clear that the optimal control method is more accurate for small values of $N$. It seems that the errors for $N=16$, in case of OC method, are caused by machine error. The numerical solutions are computed by two methods and summarized in Figure 1, and it seems that our method compared very well with those obtained via the collocation method.

Example 4.2. Consider the following VIE:

$$
x(s)=1+\sin ^{2}(s)-3 \int_{0}^{s} \sin (s-t) x^{2}(t) d t, \quad s \in[0,10]
$$

The exact solution is $x(s)=\cos (s)$. This example can be solved by using the proposed OC method. The numerical results together with computational effort of errors in boundaries and CPU time/iteration are given in Table 2.

The computational efforts presented here proved that we could rearrange in a way to avoid the rounding errors in collocation and reducing the CPU time/iteration processes. Furthermore, this rearrangement of the computation leads to a much more accurate and robust method. 
Table 2: Observed results for Example 4.2.

\begin{tabular}{lccc}
\hline Methods & $N$ & CPU time/iteration & Errors in boundaries \\
\hline & 8 & 0.74 sec. & $3.7 \mathrm{E}-02$ \\
Chebyshev collocation & 12 & $1.43 \mathrm{sec}$. & $1.9 \mathrm{E}-04$ \\
method & 16 & $1.89 \mathrm{sec}$. & $2.0 \mathrm{E}-06$ \\
& 20 & $2.01 \mathrm{sec}$. & $7.7 \mathrm{E}-07$ \\
& 24 & $2.11 \mathrm{sec}$. & $3.1 \mathrm{E}-09$ \\
\hline \multirow{3}{*}{ Optimal control method } & 8 & $0.03 \mathrm{sec}$. & $1.1 \mathrm{E}-05$ \\
& 12 & $0.28 \mathrm{sec}$. & $7.6 \mathrm{E}-07$ \\
& 16 & $0.86 \mathrm{sec}$. & $5.0 \mathrm{E}-09$ \\
& 20 & $1.01 \mathrm{sec}$. & $3.2 \mathrm{E}-11$ \\
& 24 & $1.19 \mathrm{sec}$. & $4.1 \mathrm{E}-13$ \\
\hline
\end{tabular}

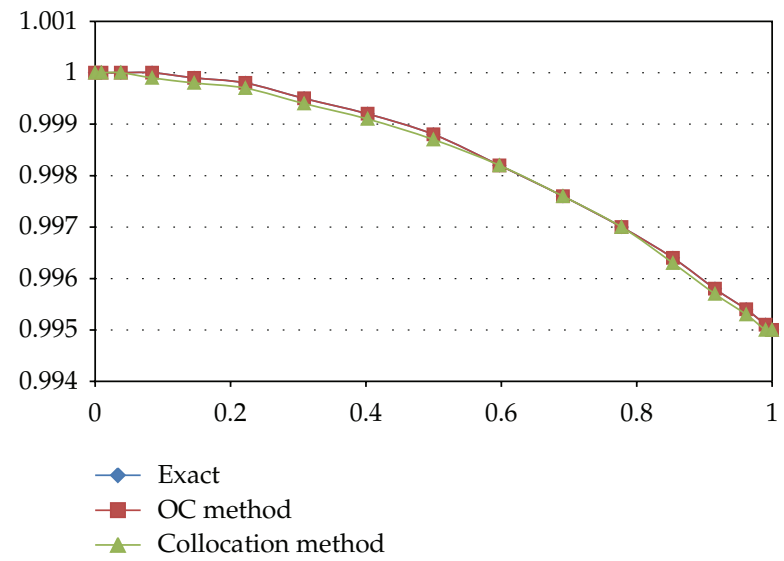

Figure 2: Observed results for Example $4.2(N=16)$.

In Figure 2, the proposed OC method shows the observed results for Example 4.2 for $N=16$. It seems also that OC method is more accurate than collocation methods.

Example 4.3. Consider the nonlinear Volterra-Fredholm integral equation

$$
x(s)=y(s)+\int_{0}^{s}(s-t) x^{2}(t) d t+\int_{0}^{1}(s+t) x(t) d t
$$

with $y(s)=(-1 / 30) s^{6}+(1 / 3) s^{4}-s^{2}+(5 / 3) s-(5 / 4)$. We applied the OC method presented in this paper and solved (4.3). The computational results together with the exact solution $x(s)=s^{2}-2$ are given in Figure 3. 


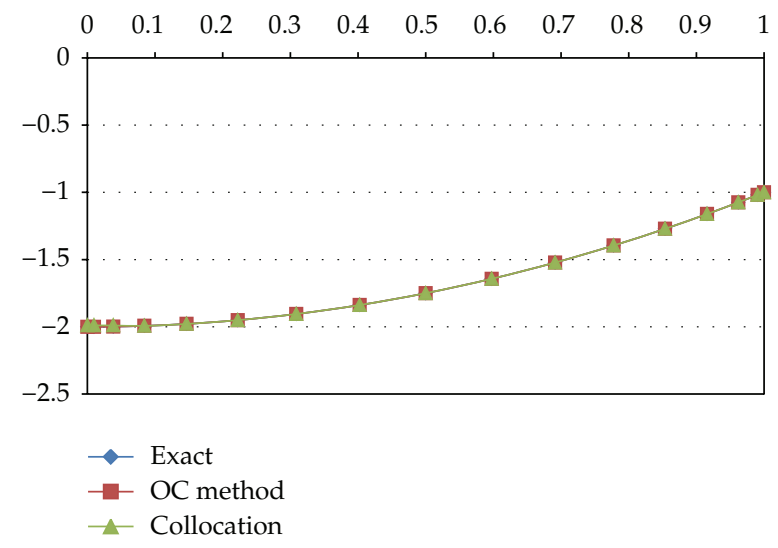

Figure 3: Observed results for Example $4.3(N=16)$.

\section{Conclusion}

In this paper, the optimal control method is introduced to simplify the implementation of general nonlinear integral equations of the second kind. We have shown, in numerical examples, that this method is fast and gains better results compared with collocation method. The important thing to note is that the control-state constraint is satisfied everywhere. Furthermore, the structure of the optimal control agrees with the results obtained in [16].

\section{References}

[1] A. K. Khamis and M. A. El-Ameen, "Volterra-fredholm integral equation with carlman kernel in position and time," Journal of Engineering and Applied Sciences, vol. 4, no. 5-6, pp. 288-294, 2009.

[2] E. V. Kovalenko, "Some approximate methods for solving integral equations for mixed problems," Journal of Applied Mathematics and Mechanics, vol. 53, no. 1, pp. 85-92, 1989.

[3] B. I. Smetanin, "On an integral equation of axisymmetric problems for an elastic body containing an inclusion," Journal of Applied Mathematics and Mechanics, vol. 55, no. 3, pp. 371-375, 1991.

[4] M. A. El-Ameen, "Approximate solutions of nonlinear Fredholm integral equations," Applied Mathematics \& Information Sciences, vol. 5, no. 1, pp. 122-131, 2011.

[5] P. Linz, Analytical and Numerical Methods for Volterra Equations, vol. 7, Society for Industrial and Applied Mathematics, Philadelphia, Pa, USA, 1985.

[6] K. Maleknejad and M. Hadizadeh, "The numerical analysis of Adomian's decomposition method for nonlinear Volterra integral and integro-differential equations," International Journal of Engineering Science, vol. 8, no. 2a, pp. 33-48, 199.

[7] A.-M. Wazwaz, A First Course in Integral Equations, World Scientific, Singapor, 1997.

[8] F. G. Tricomi, Integral Equations, Dover, New York, NY, USA, 1982.

[9] A. A. Daşcioglu, "A Chebyshev polynomial approach for linear Fredholm-Volterra integro-differential equations in the most general form," Applied Mathematics and Computation, vol. 181, no. 1, pp. 103-112, 2006.

[10] H. Brunner, Collocation Methods for Volterra Integral and Related Functional Differential Equations, vol. 15, Cambridge University Press, Cambridge, UK, 2004.

[11] L. M. Delves and J. L. Mohamed, Computational Methods for Integral Equations, Cambridge University Press, Cambridge, UK, 1985.

[12] K. Maleknejad and H. Derili, "The collocation method for Hammerstein equations by Daubechies wavelets," Applied Mathematics and Computation, vol. 172, no. 2, pp. 846-864, 2006.

[13] K. Maleknejad, M. Karami, and M. Rabbani, "Using the Petrov-Galerkin elements for solving Hammerstein integral equations," Applied Mathematics and Computation, vol. 172, no. 2, pp. 831-845, 2006. 
[14] S. Yalcinbas, "Taylor polynomial solutions of nonlinear Volterra-Fredholm integral equations," Applied Mathematics and Computation, vol. 127, no. 2-3, pp. 195-206, 2002.

[15] S. Yousefi and M. Razzaghi, "Legender wavelet method for the nonlinear Volterra- Fredholm integral equations," Mathematics and Computers in Simulation, vol. 70, no. 1, pp. 1-8, 2005.

[16] Y. Ordokhani and M. Razzaghi, "Solution of nonlinear Volterra-Fredholm-Hammerstein integral equations via a collocation method and rationalized Haar functions," Applied Mathematics Letters, vol. 21, no. 1, pp. 4-9, 2008.

[17] E. Babolian, F. Fattahzadeh, and E. G. Raboky, "A Chebyshev approximation for solving nonlinear integral equations of Hammerstein type," Applied Mathematics and Computation, vol. 189, no. 1, pp. 641-646, 2007. 


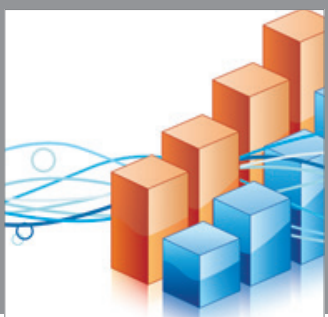

Advances in

Operations Research

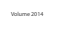

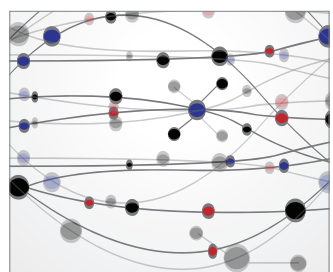

\section{The Scientific} World Journal
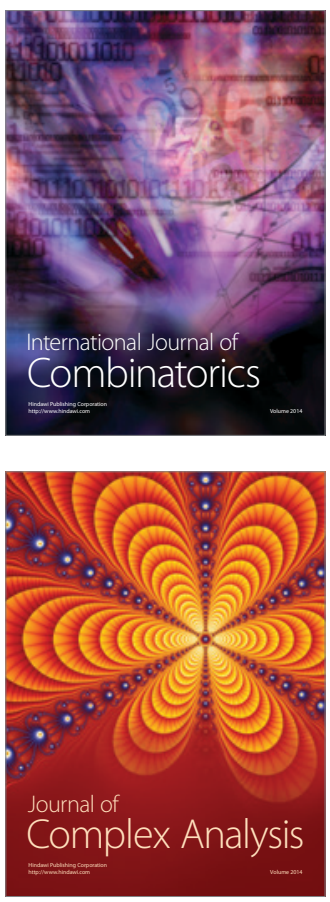

International Journal of

Mathematics and

Mathematical

Sciences
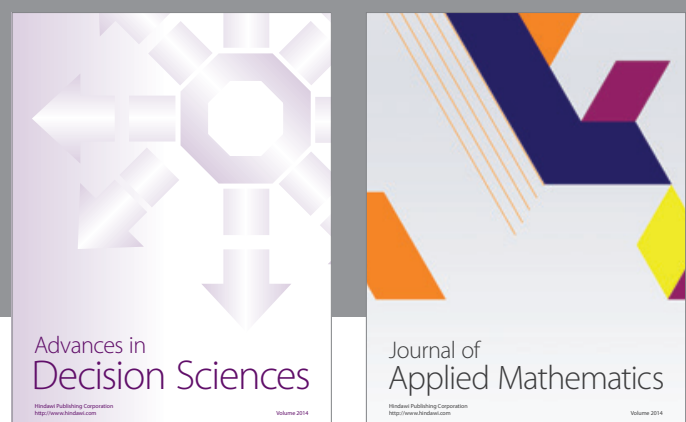

Journal of

Applied Mathematics
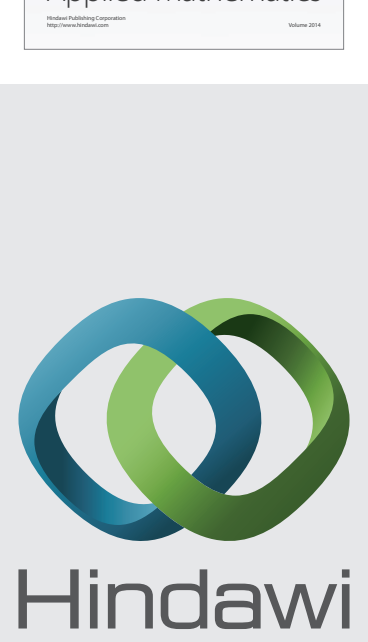

Submit your manuscripts at http://www.hindawi.com
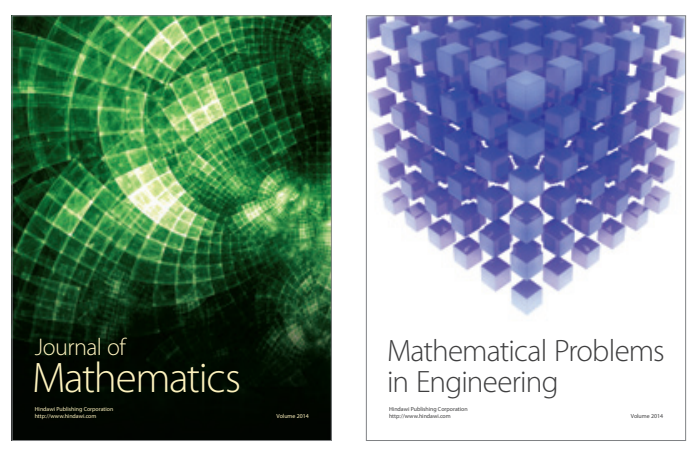

Mathematical Problems in Engineering
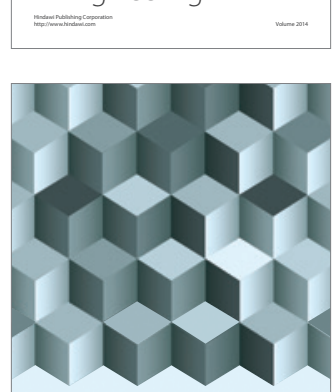

Journal of

Function Spaces
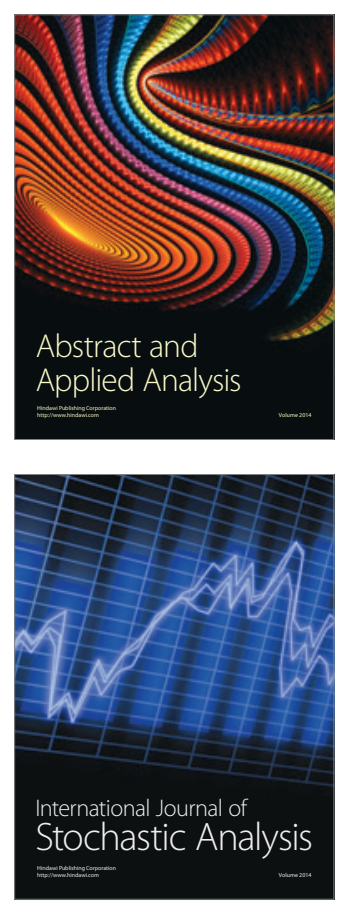

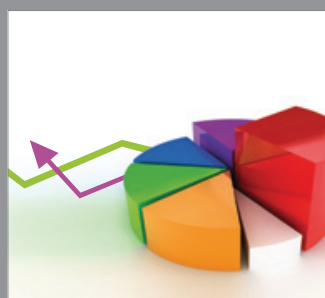

ournal of

Probability and Statistics

Promensencen
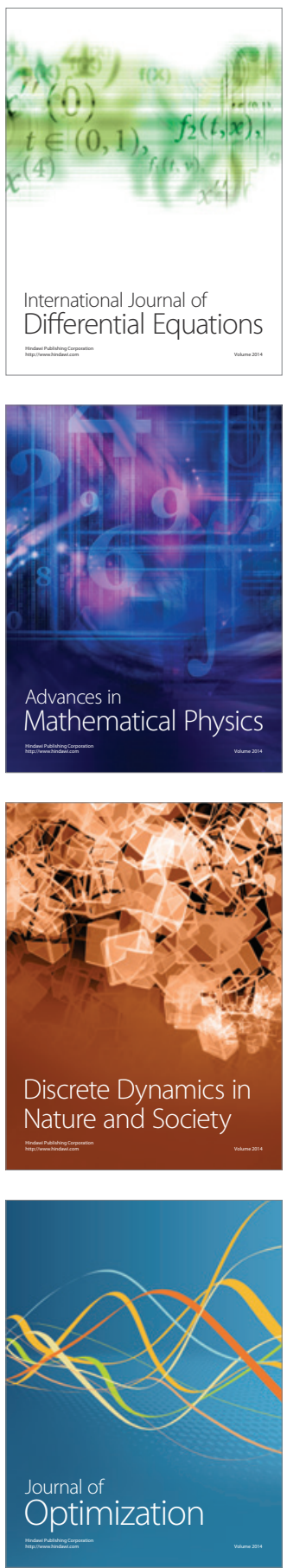